[CONTRIBUTION FROM THE CHEMICAL LABORATORY OF THE UNIVERSTTY OF CALIFORNiA.]

\title{
THE HYDROLYSIS OF ACETANILIDE.
}

BY Davtd R. MERril. AND Et,LOT Q. AdAMS.

Received June 5, 1917.

The acetylation of aniline has been studied by Menschutkin ${ }^{1}$ and by Goldschmidt and Wachs, ${ }^{2}$ but their experiments were made for the most part in glacial acetic acid or in aniline, hence the laws of aqueous solutions could not be expected to hold. The hydrolysis of acetanilide in alkaline solution has been studied by Davis. ${ }^{3}$ While the hydrolysis of other anilides and acetyl compounds in acid solution has been studied, little has been done on the hydrolysis of acetanilide, which is probably the most important. For these reasons it was thought worth while to measure the rates of reaction and equilibria concerned in a fairly dilute acid solution.

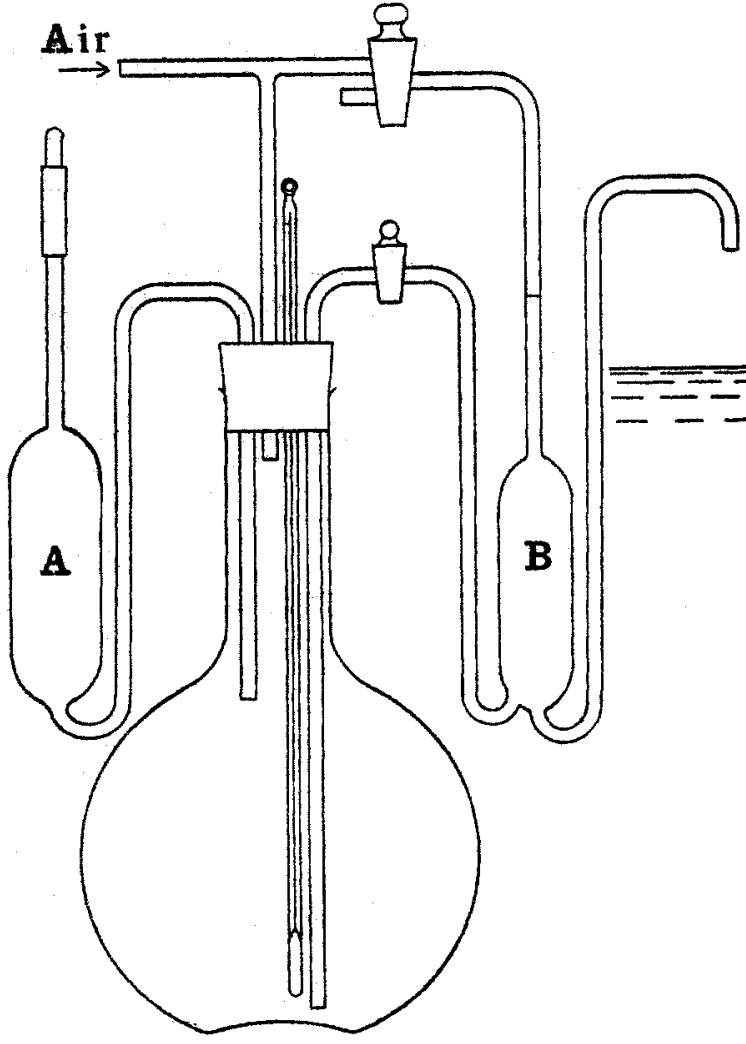

Fig. I.

Experimental Work. -The progress of the reaction was followed by determining the total acidity by titration. In the acetylation experiments solutions were made up of aniline and acetic acid, and measured out with a pipet into glass tubes which were then exhausted to about $25 \mathrm{~mm}$. and sealed. The tubes were placed in a thermostat at $100^{\circ}$, heated the requisite length of time, opened and the contents washed out and titrated. In the hydrolysis with acetic acid alone, the same amount of acetanilide was weighed out into each tube and a pipetful of acid added, since acetanilide is not

1 Menschutkin, Chem. Zentr, roo6, I, 55 I.

${ }^{2} \mathrm{H}$. Goldschmidt and C. Wachs, Z. phys. Chem., 24, 353 (1897).

3 O. M. C. Davis, I. Chem. Soc., 95, I397 (1909). 
soluble enough at room temperature to permit the making up of a solution of suitable concentration.

In the case of the hydrolysis in the presence of hydrochloric acid the reaction proceeds so much faster that it was found more convenient to use the apparatus shown in Fig. I. The water and acetanilide were placed in the flask and the hydrochloric acid in the pipet A. After the whole had come to the temperature of the bath and the acetanilide was all dissolved, the acid was blown over into the acetanilide solution and thoroughly mixed. Then by turning the stopcocks in the proper sequence a pipetful of the solution could be blown out for analysis. By always running the solution in to the same amount of cold water and titrating immediately, fair uniformity of the conditions was obtained. Because of the method of calculation used, the actual time from the beginning was not so important as uniformity in the measurement of the time of drawing the sample.

Method of Calculation.-We shall consider first the simplest case, namely, the hydrolysis in the presence of hydrochloric acid. Since at the concentration of acid present throughout the experiments the ionization of acetic acid and the "hydrolysis" of aniline hydrochloride are largely repressed-while the concentration of acetanilide hydrochloride is negligible - the ionic equation for the reaction studied is

$$
\mathrm{C}_{6} \mathrm{H}_{5} \mathrm{NHCOCH}_{3}+\mathrm{H}_{2} \mathrm{O}+\mathrm{H}^{+} \longrightarrow \mathrm{C}_{6} \mathrm{H}_{5} \mathrm{NH}_{3}++\mathrm{CH}_{3} \mathrm{COOH} \text {. }
$$

As the concentration of the water (more correctly its activity) does not change materially, the reaction may be expected to have the character of a bimolecular reaction, the rate being proportional to the product of the concentrations of acetanilide and hydrogen ion. (Under the experimental conditions the reverse reaction is entirely negligible.) The equations for the progress of such a reaction are

$$
\begin{gathered}
d x=k_{2}(a-x)(b-x) d t \\
2.303 \log \frac{b-x}{a-x}=(b-a) k_{2} t+2.303 \log \frac{b}{a}
\end{gathered}
$$

where $a, b$ and $x$ are, respectively; ${ }^{1}$ the acid and acetanilide originally present, and the amount of each which has been used up at time $t$. The correction that must be made for the hydrogen ion furnished by the acetic acid and by the aniline ion is as follows: The ionization constant of acetic $\operatorname{acid}^{2}$ at $100^{\circ}$ is $\mathrm{I} . \mathrm{II} \times \mathrm{IO}^{-5}$, that of aniline $\operatorname{ion}^{3} 3 . \mathrm{I} \times \mathrm{rO}^{-4}$, so that a mixture of equivalent amounts of the two in the presence of excess mineral acid will give as much hydrogen ion as one equivalent of an acid of constant $3.2 \times 10^{-4}$. If $y$ represents the hydrogen ion so produced

1 The notation differs from that used later in the paper in the calculation of the experiments in acetic acid solution.

2 A. A. Noyes, This Journal, 30, 335 (1908).

${ }^{3}$ Determined colorimetrically, using methyl orange as an indicator. 


$$
\begin{aligned}
3.2 \times 10^{-4}(\mathrm{HA}) & =\left(\mathrm{A}^{-}\right)\left(\mathrm{H}^{+}\right) \\
3.2 \times 10^{-4}(x-y) & =y(a-x+y) \\
y & =\frac{3.2 \times 10^{-4}(x-y)}{a-x+y}
\end{aligned}
$$

or

from which $y$ may readily be calculated by approximation. Since the rate of the reaction is directly proportional to the hydrogen-ion concentration, correction for the extra hydrogen ion $y$ may be made by increasing all measured time intervals in the ratio of $\mathrm{I}+\frac{y}{a-x}$ to I. In Fig. 2, $\log \frac{b-x}{a-x}$ has been plotted against the "effective time," and from the slope $m$ of the

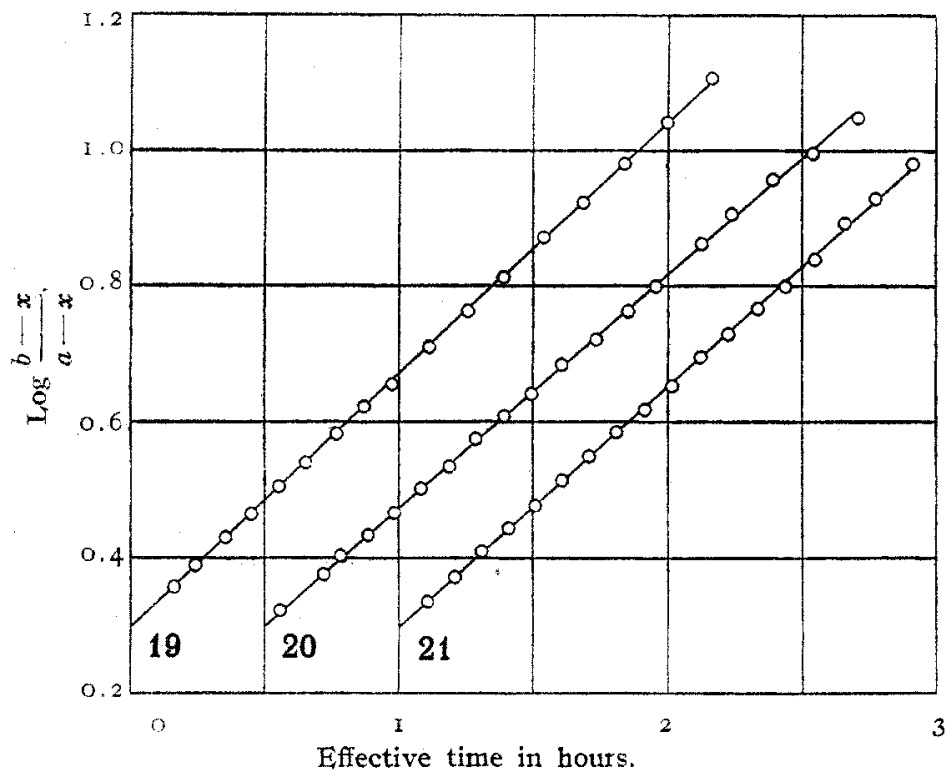

Fig. 2.

straight lines on these plots, the constant $k_{2}$ of the hydrolysis reaction has been calculated by the equation

$$
k_{2}=\frac{2.303 m}{b-a}
$$

The data and calculations are given in Tables 2 and 3 .

When the reaction takes place in acetic acid solution the speeds of the reaction in the two directions are comparable and the hydrogen ion from the aniline salt and acetic acid, instead of being a small addition to that already present, constitutes the entire hydrogen-ion concentration of the solution. In addition the aniline and acetic acid are no longer present in equivalent amounts. The equation for the progress of the reaction

$\mathrm{CH}_{3} \mathrm{COOH}+\mathrm{C}_{6} \mathrm{H}_{6} \mathrm{NH}_{3}+\rightleftarrows \mathrm{C}_{6} \mathrm{H}_{6} \mathrm{NHCOCH}_{3}+\mathrm{H}_{2} \mathrm{O}+\mathrm{H}^{+}$, 
since the activity of the water is substantially constant, takes the form

$$
d x=\left[k_{1}\left(\mathrm{CH}_{3} \mathrm{COOH}\right)\left(\mathrm{C}_{6} \mathrm{H}_{5} \mathrm{NH}_{3}{ }^{+}\right)-k_{2} x\left(\mathrm{H}^{+}\right)\right] d t
$$

where $x$ represents the concentration of acetanilide. If $a$ and $b$ be, respectively, the total acetic acid and aniline, each including acetanilide, and $x$ the acetanilide present at time $\dot{t}$, this equation can be put, according to the direction in which the reaction is proceeding, into the forms

$$
\begin{aligned}
& \frac{d x}{(a-x)(b-x)}= \\
& k_{1}\left[\frac{\left(\mathrm{CH}_{3} \mathrm{COOH}\right)\left(\mathrm{C}_{6} \mathrm{H}_{5} \mathrm{NH}_{3}+\right)}{(a-x)(b-x)}-\frac{k_{2}}{k_{1}} \frac{x\left(\mathrm{H}^{+}\right)}{(a-x)(b-x)}\right] d t \\
& -\frac{d x}{x}=k_{2}\left[\left(\mathrm{H}^{+}\right)-\frac{k_{1}}{k_{2}} \frac{\left.\left(\mathrm{CH}_{3} \mathrm{COOH}\right)\left(\mathrm{C}_{6} \mathrm{H}_{5} \mathrm{NH}_{3}^{+}\right)\right]}{x}\right] d t .
\end{aligned}
$$

TABLE I.

$\mathrm{C}_{6} \mathrm{H}_{5} \mathrm{NH}_{2}+\mathrm{CH}_{3} \mathrm{COOH}=\mathrm{C}_{8} \mathrm{H}_{3} \mathrm{NHCOCH}_{3}+\mathrm{H}_{2} \mathrm{O}$.

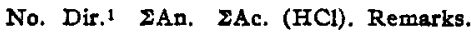

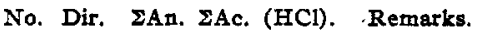

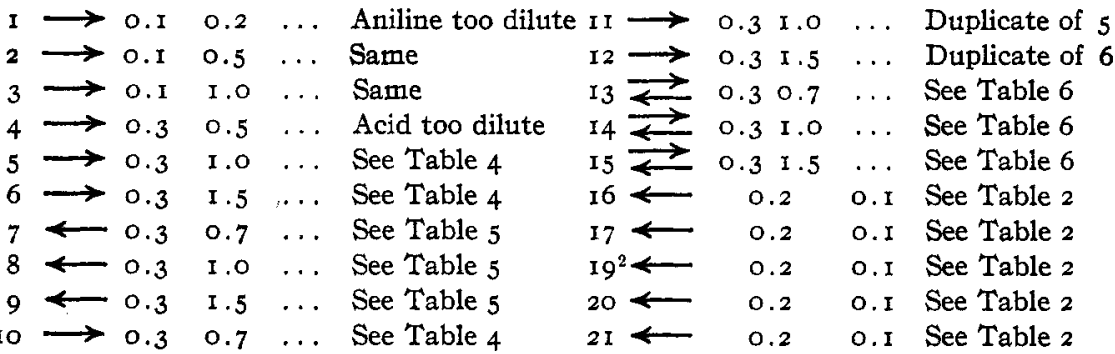

The mass-law equations for acetic acid and aniline ion take the form

$$
\begin{aligned}
& \mathrm{CH}_{3} \mathrm{COOH}=\mathrm{CH}_{3} \mathrm{COO}^{-}+\mathrm{H}^{+} \quad \mathrm{K}_{\mathrm{A}}=\frac{\left(\mathrm{CH}_{3} \mathrm{COO}^{-}\right)\left(\mathrm{H}^{+}\right)}{\left(\mathrm{CH}_{3} \mathrm{COOH}\right)} \\
& \mathrm{C}_{6} \mathrm{H}_{6} \mathrm{NH}_{3}^{+}=\mathrm{C}_{6} \mathrm{H}_{6} \mathrm{NH}_{2}+\mathrm{H}^{+} \quad \mathrm{K}_{\mathrm{B}}^{\prime}=\frac{\left(\mathrm{C}_{6} \mathrm{H}_{6} \mathrm{NH}_{2}\right)\left(\mathrm{H}^{+}\right)^{3}}{\left(\mathrm{C}_{6} \mathrm{H}_{5} \mathrm{NH}_{3}^{+}\right)}
\end{aligned}
$$

whence, since

$$
\left(\mathrm{CH}_{3} \mathrm{COO}^{-}\right)+\left(\mathrm{CH}_{3} \mathrm{COOH}\right)=a-x
$$

and

$$
\begin{gathered}
\left(\mathrm{C}_{6} \mathrm{H}_{5} \mathrm{NH}_{2}\right)+\left(\mathrm{C}_{6} \mathrm{H}_{5} \mathrm{NH}_{3}{ }^{+}\right)=b-x, \\
\frac{\left(\mathrm{CH}_{3} \mathrm{COOH}\right)}{a-x}=\frac{\left(\mathrm{H}^{+}\right)}{\overline{\mathrm{K}}_{\mathrm{A}}+\left(\mathrm{H}^{+}\right)} \\
\frac{\left(\mathrm{C}_{6} \mathrm{H}_{6} \mathrm{NH}_{8}+\right)}{b-x}=\frac{\left(\mathrm{H}^{+}\right)}{\mathrm{K}_{\mathrm{B}}^{\prime}+\left(\mathrm{H}^{+}\right)} .
\end{gathered}
$$

1 The arrow shown is to be substituted for the $=$ sign in the equation.

${ }^{2}$ Series 18 was lost because the acetanilide was not all in solution.

8 This is not the usual $\mathrm{K}_{B}$ of aniline, but its reciprocal multiplied by the dissociation constant of water. As no data were available for this temperature, we have determined $\mathrm{K}_{\mathrm{B}}^{\prime}$ at $100^{\circ}$ for aniline to be $3 . \mathrm{I} \times \mathrm{IO}^{-4}$. 


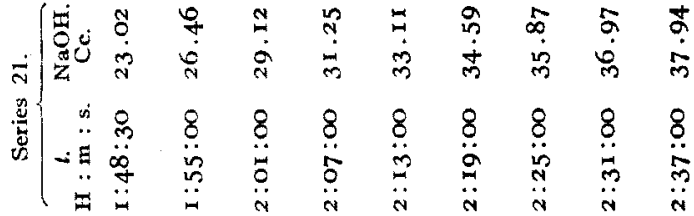

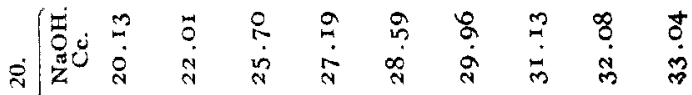

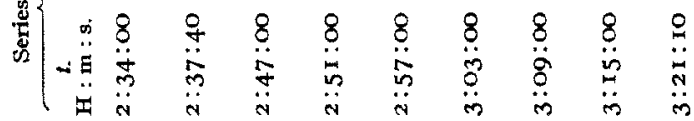

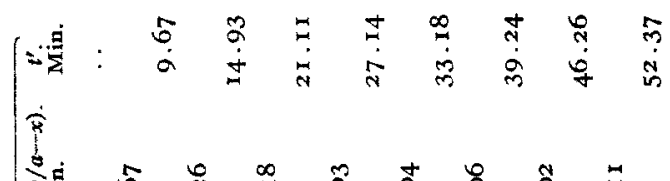

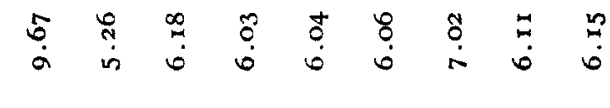

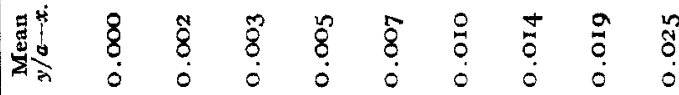

恶

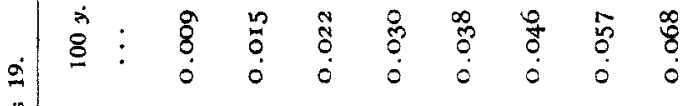

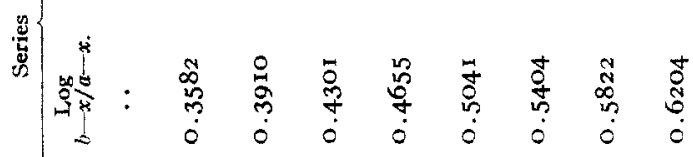

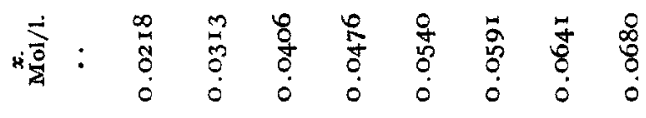

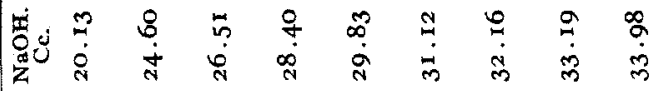

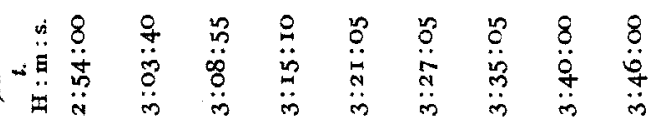

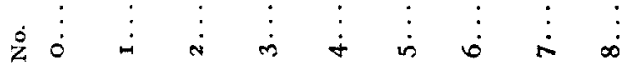




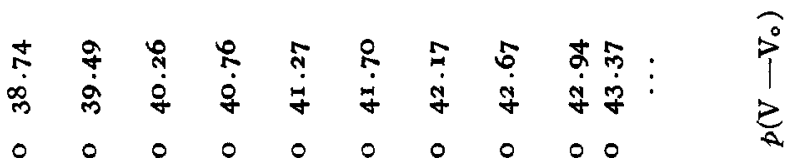

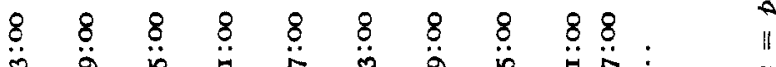

学

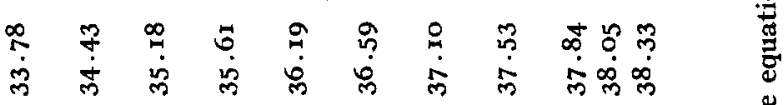

88888888888 요 8

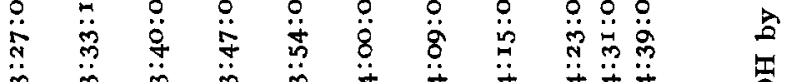

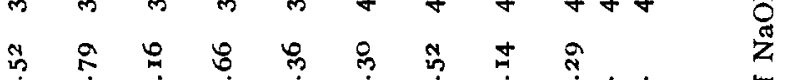

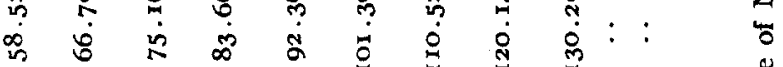

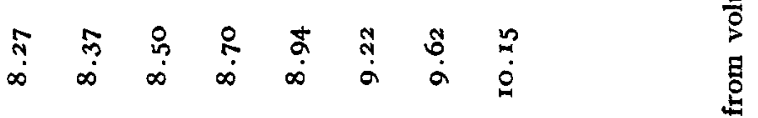

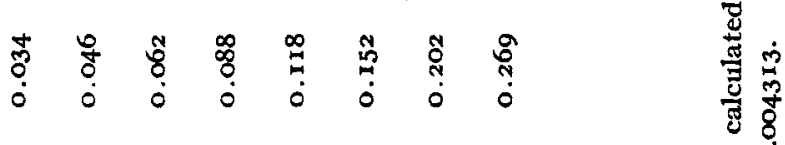

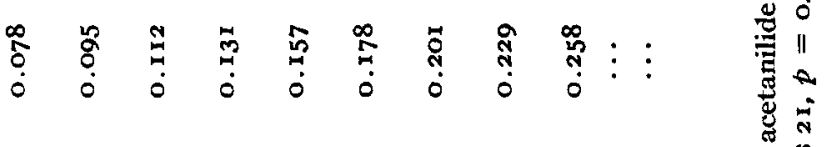

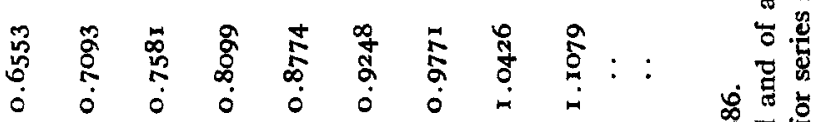

₹ : की

荥

$\begin{array}{llllllllll}8 & 8 & 8 & 8 & 8 & 8 & 8 & 8 & 8 & \end{array}$

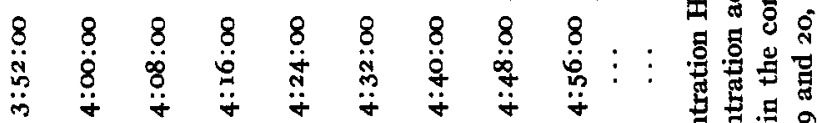

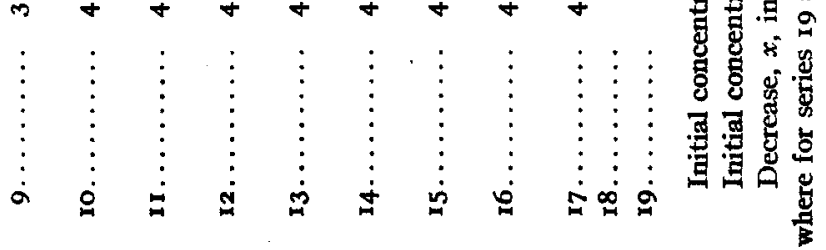


TABLE 3 .

\begin{tabular}{|c|c|c|c|c|c|}
\hline ries No. & $\begin{array}{l}\text { Slope, } \\
\text { recip. irr. }\end{array}$ & $\underset{1 / \mathrm{mol} \times \mathrm{hr} .}{k_{k}}$ & Temp. obs. & $\begin{array}{l}\text { Barometer, } \\
\text { in. (of } \mathbf{~ H g} \text {. }\end{array}$ & Temp. calc. \\
\hline 19 & 0.3725 & 8.64 & $100.3^{\circ}$ & 29.97 & $100.05^{\circ}$ \\
\hline $20 \ldots$ & 0.349 & 8.10 & $99.7^{\circ}$ & $29.8 \mathrm{I}$ & $99.90^{\circ}$ \\
\hline $2 x \ldots \ldots \ldots \ldots \ldots \ldots$ & .0 .352 & 8.17 & $99.5^{\circ}$ & 29.45 & $99.55^{\circ}$ \\
\hline & . & 8.30 & $99.83^{\circ}$ & . & $99.83^{\circ}$ \\
\hline Reduced & .. & 8.43 & & $\cdots$ & $100.00^{\circ}$ \\
\hline
\end{tabular}

Series $I 6$ and $I 7$ were performed in an oil thermostat and gave for $k_{2}, 7.46$ and 7.92 , respectively. An investigation into the cause of this discrepancy showed that the thermostat regulator was not operating satisfactorily. The subsequent experiments were performed in a boiling water bath and the result reduced to $100^{\circ}$, assuming a $2^{1 / 2}$-fold increase for $10^{\circ}$.

Substituting in Equations $\left(\mathrm{II}_{1}\right)$ and $\left(\mathrm{II}_{2}\right)$, since $k_{1} / k_{2}=\mathrm{K}$, the equilibrium constant for the acetylation of aniline (determined in separate experiments, see Table 6),

$$
\begin{aligned}
& \frac{d x}{(a-x)(b-x)}=k_{1}\left[\frac{\left(\mathrm{H}^{+}\right)^{2}}{\left(\mathrm{~K}_{\mathrm{A}}+\left(\mathrm{H}^{+}\right)\right)\left(\mathrm{K}_{\mathrm{B}}^{\prime}+\left(\mathrm{H}^{+}\right)\right)}-\frac{\mathrm{I}}{\mathrm{K}} \frac{x\left(\mathrm{H}^{+}\right)}{(a-x)(b-x)}\right] d t\left(\mathrm{VI}_{1}\right) \\
& -\frac{d x}{x}=k_{2}\left[\mathrm{H}^{+}-\mathrm{K} \frac{(a-x)(b-x)}{x} \frac{\left(\mathrm{H}^{+}\right)^{2}}{\left(\mathrm{~K}_{\mathrm{A}}+\left(\mathrm{H}^{+}\right)\right)\left(\mathrm{K}_{\mathrm{B}}^{\prime}+\left(\mathrm{H}^{+}\right)\right)}\right] d t\left(\mathrm{VI}_{2}\right)
\end{aligned}
$$

or

$$
\begin{aligned}
\frac{2.303}{b-a} \log \left(\frac{b-x}{a-x}\right)=k_{1} \int & {\left[\frac{\left(\mathrm{H}^{+}\right)^{2}}{\left(\mathrm{~K}_{\mathrm{A}}+\left(\mathrm{H}^{+}\right)\right)\left(\mathrm{K}_{\mathrm{B}}^{\prime}+\left(\mathrm{H}^{+}\right)\right)}-\right.} \\
& \left.\frac{\mathrm{I}}{\mathrm{K}} \frac{x\left(\mathrm{H}^{+}\right)}{(a-x)(b-x)}\right] d t+\frac{2.303}{b-a} \log \frac{b}{a} .
\end{aligned}
$$

$2.303 \log \frac{x_{0}}{x}=$

$$
k_{2} \int\left[\left(\mathrm{H}^{+}\right)-\mathrm{K} \frac{(a-x)(b-x)}{x} \frac{\left(\mathrm{H}^{+}\right)^{2}}{\left(\mathrm{~K}_{\mathrm{A}}+\left(\mathrm{H}^{+}\right)\right)\left(\mathrm{K}_{\mathrm{B}}^{\prime}+\left(\mathrm{H}^{+}\right)\right)}\right] d t . \quad\left(\mathrm{VII}_{2}\right)
$$

Now from (IVa), (IV $b),(\mathrm{V} a),(\mathrm{V} b)$, since in the absence of other electrolytes

$$
\begin{array}{r}
\left(\mathrm{H}^{+}\right)=\left(\mathrm{CH}_{3} \mathrm{COO}^{-}\right)-\left(\mathrm{C}_{6} \mathrm{H}_{5} \mathrm{NH}_{3}+\right), \\
\left(\mathrm{H}^{+}\right)=\frac{\mathrm{K}_{\mathrm{A}}}{2}\left(\sqrt{\mathrm{I}+\left(\frac{4 \mathrm{~K}_{\mathrm{B}}^{\prime}}{\mathrm{K}_{\mathrm{A}}}-2\right) \frac{a-x}{b-x+\mathrm{K}_{\mathrm{B}}^{\prime}+\left(\mathrm{H}^{+}\right)}+\left(\frac{a-x}{b-x+\mathrm{K}_{\mathrm{B}}^{\prime}+\left(\mathrm{H}^{+}\right)}\right)^{2}}\right. \\
\left.+\frac{a-x}{b-x+\mathrm{K}_{\mathrm{B}}^{\prime}+\left(\mathrm{H}^{+}\right)}-\mathrm{I}\right) \quad \text { (IX) }
\end{array}
$$

whence $\left(\mathrm{H}^{+}\right)$may be calculated by approximation. Then by plotting, respectively, $\log \left(\frac{b-x}{a-x}\right)$ and $-\log x$ against the "effective" values for the time, obtained by multiplying every measured time interval by the mean value of the respective expression in square brackets, straight lines should 
be obtained from whose slopes, $m_{1}, m_{2}$, the reaction velocity constants can be calculated, as in the earlier case of hydrolysis by hydrochloric acid, by means of the equations

$$
k_{1}=\frac{2.303 m_{1}}{b-a} \quad k_{2}=2.303 m_{2} .
$$

The results are shown plotted in Figs. 3 and 4 ; the data and calculations will be found in Tables 4,5 and 6 . The concordance is not all that could be desired, but since the equilibrium experiments indicated side

TABLE 4.

\begin{tabular}{|c|c|c|c|c|c|c|c|}
\hline Series No. & $\begin{array}{l}\text { Time. } \\
\text { Hrs. }\end{array}$ & $\underset{\mathrm{mol} / \mathrm{s}}{x}$ & $\begin{array}{c}\log \\
(a-x) /(b-x) .\end{array}$ & $\begin{array}{c}104\left(\mathrm{H}^{+}\right) \\
\mathrm{mol} / 1 .\end{array}$ & $\begin{array}{c}\text { Factor. } \\
{[] \text {. }}\end{array}$ & $\underset{\text { Frs. }}{\Delta i \times[]_{\text {mean }}}$ & $\begin{array}{l}t^{\prime} \\
\text { Hrs. }\end{array}$ \\
\hline $6-2$ & 6.5 & 0.01476 & 0.720 & I. 598 & 0.312 & $3 \cdot 56$ & $\cdots$ \\
\hline 3 & 18.0 & 0.02970 & 0.739 & I. 646 & 0.309 & 3.21 & 3.56 \\
\hline 4 & 28.5 & 0.04877 & 0.768 & 1.712 & 0.302 & 6.04 & 6.77 \\
\hline 5 & $4^{8.75}$ & 0.06955 & $0.80 \mathrm{I}$ & I.798 & 0.293 & 6.60 & $\mathrm{I2} .8 \mathrm{I}$ \\
\hline 6 & 72.0 & 0.0899 & 0.836 & I .895 & 0.276 & 6.88 & I9.4I \\
\hline 7 & 98.0 & 0.1107 & 0.879 & 2.018 & 0.254 & & 26.29 \\
\hline $5^{-1}$ & 1.92 & 0.00329 & 0.530 & 1.221 & 0.2579 & I. 42 & $\cdots$ \\
\hline 2 & $7 \cdot 42$ & 0.00776 & 0.535 & 1.229 & 0.2575 & 2.93 & $I .42$ \\
\hline 3 & 18.92 & $0.0206 \mathrm{I}$ & 0.550 & I. 255 & 0.2528 & 2.63 & $4 \cdot 35$ \\
\hline 4 & 29.42 & 0.02990 & 0.560 & I.274 & 0.2490 & 4.99 & 6.98 \\
\hline 5 & 49.67 & 0.04432 & 0.580 & I. 309 & 0.2444 & 5.60 & II.97 \\
\hline 6 & 72.92 & 0.0580 & 0.598 & I. 343 & $0.237^{\circ}$ & 6.04 & $17 \cdot 57$ \\
\hline 7 & 98.92 & 0.0717 & 0.618 & I. 384 & 0.2274 & & $23.6 \mathrm{I}$ \\
\hline $10-1$ & 26 & 0.01778 & 0.386 & 0.998 & 0.2086 & $4 \cdot 36$ & . \\
\hline 2 & 47 & 0.02381 & 0.392 & 1.006 & 0.2059 & 9.98 & $4 \cdot 36$ \\
\hline 3 & 97 & 0.04504 & $0.4^{1} 4$ & 1.037 & 0.1933 & $17.4 \mathrm{I}$ & 14.34 \\
\hline 4 & 193 & 0.0729 & 0.446 & 1.086 & 0.1696 & 24.89 & $31 \cdot 75$ \\
\hline 5 & $36 r$ & 0.1027 & 0.490 & I. I 53 & 0.1266 & 32.58 & 56.64 \\
\hline 6 & 697 & 0.1265 & $0.53 \mathrm{I}$ & 1.222 & 0.0673 & & 89.22 \\
\hline
\end{tabular}




\begin{tabular}{|c|c|c|c|c|c|c|c|}
\hline \multirow[b]{2}{*}{ Series No. } & \multirow[b]{2}{*}{$\begin{array}{l}\text { Time. } \\
\text { Hours. }\end{array}$} & \multicolumn{4}{|c|}{ TABLE 5.} & \multirow[b]{2}{*}{$104 \Delta i \times[]_{\text {mean }}$} & \multirow{2}{*}{$\begin{array}{c}10 \mathrm{u}^{\prime} \\
\mathrm{mol} \times \mathrm{br} . / \mathrm{l} .\end{array}$} \\
\hline & & $\underset{\mathrm{mol} / \mathrm{l}}{x}$ & $1+\log x$ & $\begin{array}{c}10^{4}\left(\mathrm{H}^{+}\right) \\
\mathrm{mol} / 1\end{array}$ & $\begin{array}{l}104[] \\
\mathrm{mol} / 1\end{array}$ & & \\
\hline 9-I & 46.5 & $0.232 \mathrm{r}$ & 0.3657 & 4.188 & 3.902 & $86 . \mathrm{I}$ & $\cdots$ \\
\hline 2 & 70.5 & 0.2189 & 0.3402 & 3.604 & 3.268 & 143.7 & $86 . \mathrm{I}$ \\
\hline 3 & 118.5 & 0.2022 & 0.3058 & 3.110 & 2.716 & I 21.2 & 229.8 \\
\hline 4 & $\mathrm{I} 64.5$ & 0.1956 & 0.2914 & 2.968 & $2.55^{2}$ & I 81.5 & 351.0 \\
\hline 5 & 238.5 & 0.1867 & 0.2711 & 2.800 & $2 \cdot 354$ & & 532.5 \\
\hline $8-1$ & 47 & 0.2407 & $0.3^{815}$ & 3.246 & $3 \cdot 105$ & 68,1 & $\cdots$ \\
\hline 2 & 71 & 0.2267 & 0.3554 & 2.746 & 2.573 & $\mathrm{II}_{3} \cdot 3$ & $68 . \mathrm{I}$ \\
\hline 3 & II & 0.2092 & 0.3206 & 2.359 & $2.15 \mathrm{I}$ & 93.6 & 181.4 \\
\hline 4 & 165 & 0.1958 & 0.2918 & 2.150 & I.916 & $92 \cdot 3$ & 275.0 \\
\hline 5 & 215 & 0.1860 & 0.2695 & 2.030 & 1.776 & & $367 \cdot 3$ \\
\hline $7-1$ & 49 & 0.2546 & 0.4059 & $2.82 \mathrm{I}$ & 2.763 & 58.6 & \\
\hline 2 & 73 & 0.2373 & 0.3753 & 2.196 & 2.117 & IOO.I & 58.6 \\
\hline 3 & I 25 & 0.2181 & $0.33^{87}$ & I. 832 & 1.730 & 68.6 & 158.7 \\
\hline 4 & 167 & 0.2039 & 0.3094 & 1.662 & I. 543 & $109 \cdot 3$ & $227 \cdot 3$ \\
\hline 5 & $24 \mathrm{I}$ & 0.1912 & 0.2815 & I. 547 & I. $4^{\text {I } 4}$ & & 336.6 \\
\hline
\end{tabular}

\begin{tabular}{|c|c|c|c|c|c|c|}
\hline \multirow[b]{2}{*}{$\begin{array}{l}\text { Series } \\
\text { No. }\end{array}$} & \multicolumn{6}{|c|}{ TABLE 6.} \\
\hline & $\begin{array}{c}2 \mathrm{CH}_{2} \mathrm{CO}_{2} \mathrm{H} \\
a \\
\mathrm{~mol} / \mathrm{l} .\end{array}$ & $\begin{array}{c}\Sigma \mathrm{C}_{6} \mathrm{H}_{3} \mathrm{NH}_{2} \\
b \\
\mathrm{~mol} / 1 .\end{array}$ & $\begin{array}{l}\text { Slope } \\
m \text { me } \\
\text { recip. br. }\end{array}$ & $\begin{array}{c}2.303 \mathrm{~m} / a-b \\
k_{2} \\
1 / \mathrm{mol} \times \mathrm{hr} .\end{array}$ & $\stackrel{1 / \operatorname{mol}_{2} \times \mathrm{hr} .}{k_{2}}$ & K. \\
\hline 6 & 1.437 & 0.2860 & 0.00604 & 0.01209 & & \\
\hline 5 & 0.963 & 0.2862 & 0.00374 & 0.01272 & & \\
\hline 10 & 0.672 & 0.2870 & $\begin{array}{c}0.001974 \\
1 / \mathrm{mol} \times \mathrm{hr}\end{array}$ & 0.01180 & & \\
\hline 9 & I. 429 & 0.2860 & 2.65 & & 6.10 & \\
\hline 8 & 0.954 & 0.2862 & 3.09 & & 7.12 & \\
\hline 7 & 0.670 & 0.2870 & 3.65 & & $8.4 \mathrm{I}$ & \\
\hline I5 & I. 442 & 0.2860 & & & & $7.92 \times 10^{-4}$ \\
\hline $1_{4}$ & 0.963 & 0.2862 & & & & $8.78 \times 10^{-1}$ \\
\hline 13 & 0.671 & 0.2870 & & & & $9.32 \times 10^{-4}$ \\
\hline Extr & apolated $t$ & $O$ conc. $O$ & of $\mathrm{CH}_{3} \mathrm{CO}_{2} \mathrm{H}$ & & & $10.54 \times 10^{-4}$ \\
\hline
\end{tabular}

reactions 1 such as the formation of diphenylamine (which was in fact shown qualitatively to be formed)-a trigh degree of concordance could hardly be expected. There is the possibility that in addition to the

1 All the tubes used in the quantitative work developed a more or less pronounced magenta color, even though they were exhausted down to $25 \mathrm{~mm}$. before sealing. Subse- 
catalytic hydrolysis of acetanilide by hydrogen ion and by hydroxyl ion $\mathrm{C}_{6} \mathrm{H}_{5} \mathrm{NHCOCH}_{3}+\mathrm{H}^{+}+\mathrm{H}_{2} \mathrm{O}=\mathrm{C}_{6} \mathrm{H}_{5} \mathrm{NH}_{3}++\mathrm{HOCOCH}_{3}$ $\mathrm{C}_{6} \mathrm{H}_{5} \mathrm{NHCOCH}_{3}+\mathrm{OH}^{-}=\mathrm{C}_{6} \mathrm{H}_{5} \mathrm{NH}_{2}+\mathrm{OCOCH}_{8}-$ direct hydrolysis by water may occur

\section{$\mathrm{C}_{6} \mathrm{H}_{5} \mathrm{NHCOCH}_{3}+\mathrm{H}_{2} \mathrm{O}=\mathrm{C}_{6} \mathrm{H}_{5} \mathrm{NH}_{2}+\mathrm{HOCOCH}_{3}$}

at a rate which is negligible in solutions containing an appreciable amount of strong acid or alkali and yet large enough to constitute the greater part of the hydrolysis in neutral solution.

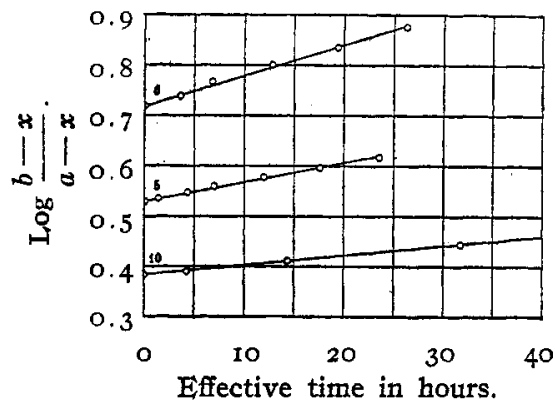

Fig. 3.

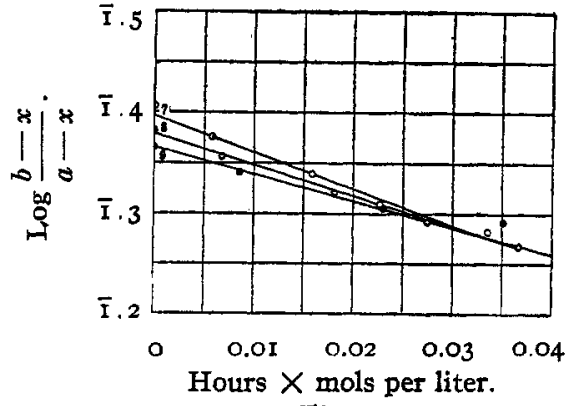

Fig. 4.

The results of the experiments on the hydrolysis in the presence of hydrochloric acid agree within the constancy of the temperature, about $0.2^{\circ}$, corresponding to $2 \%$ on the value of $k_{2}$, so that the mean value, reduced to $100^{\circ}$, may be taken as $8.4 \pm 0.1$. This result is to be compared with the corresponding constant found by Davis $^{1}$ for the hydrolysis by alkali, namely 3.2 (at the boiling point of tenth molal solution). The most probable value of the acetylation constant $k_{1}$ may be found by extrapolating ${ }^{2}$ the equilibrium constant $\mathrm{K}$ to zero concentration of acetic acid, and multiplying by $k_{2}$. The values thus obtained are $\mathrm{K}=10.5 \times \mathrm{IO}^{-4}, k_{1}=0.89 \times \mathrm{IO}^{-2}$.

\section{Summary.}

I. The hydrolysis of acetanilide by hydrochloric acid proceeds in accordance with the equation

$$
\mathrm{C}_{6} \mathrm{H}_{5} \mathrm{NHCOCH}_{3}+\mathrm{H}^{+}+\mathrm{H}_{2} \mathrm{O}=\mathrm{C}_{6} \mathrm{H}_{5} \mathrm{NH}_{3}++\mathrm{HOCOCH}_{3} \text {. }
$$

2. The velocity constant at $100^{\circ}$ for the hydrolysis of acetanilide by hydrochloric acid is $8.4 \pm 0.1$ liters $^{3}$ per mol per hour. (Davis found 3.2 for hydrolysis by alkali.)

quent experiment has shown that continued boiling out under this pressure entirely prevents the development of color, but to do so without change in concentration seemed impracticable.

1 Davis, Loc. cit. Both constants are in liters per mol per hour.

2 Direct extrapolation of the values of $k_{1}$ gives a result consistent with this, but the curvature of the graph makes extrapolation inconclusive.

${ }^{3}$ Concentrations are stated in mols per cubic decimeters at $100^{\circ}$. 
3. The most probable value (obtained by extrapolation to zero concentration of acetic acid) of the equilibrium constant (omitting the concentration of the water) for the reaction as written above is $950(=1 / 10.5 \times$ $\left.10^{-4}\right)$; this corresponds to a velocity constant for the acetylation of 0.0089 liters per mol per hour.

In conclusion we wish to express our gratitude to Prof. H. C. Biddle for his many helpful suggestions.

Barkelay, CaLrFornia.

[CONTRIBUTION FROM THE DEPARTMENT OF CHEMISTRY OF THE UNIVERSITY OF NORTH Carolina.]

\section{THE ATOMIC WEIGHT OF ZIRCONIUM.}

By Francis P. Venable and Jaygs M. BeLL.

Received June 8, 1917.

As the results of our work involve a considerable change in the atomic weight of zirconium, it is well to give a brief review of the previous determinations of this constant.

TABLE I. ${ }^{1}$

Previous Determinations of the Atomic Weight of Zirconium.

\begin{tabular}{|c|c|c|c|c|c|}
\hline & Date. & Chemist. & Ratio. & $\begin{array}{l}\text { Number of } \\
\text { analyses. }\end{array}$ & $\begin{array}{l}\text { A tomic } \\
\text { weight. }\end{array}$ \\
\hline & 1825 & Berzelius $^{2}$ & $\mathrm{Zr}\left(\mathrm{SO}_{4}\right)_{2}: \mathrm{ZrO}_{2}$ & 6 & 89.46 \\
\hline & $188 \mathrm{I}$ & Weibull $^{3}$ & $\mathrm{Zr}\left(\mathrm{SO}_{4}\right)_{2}: \mathrm{ZrO}_{2}$ & 7 & 89.54 \\
\hline 3. & 1889 & Bailey $^{4}$ & $\mathrm{Zr}\left(\mathrm{SO}_{4}\right)_{2}: \mathrm{ZrO}_{2}$ & 8 & 90.65 \\
\hline $4 \ldots \ldots \ldots$ & $188 \mathrm{r}$ & Weibull $^{3}$ & $\mathrm{Zr}\left(\mathrm{SeO}_{4}\right)_{2}: \mathrm{ZrO}_{2}$ & 5 & 90.79 \\
\hline $5 \ldots \ldots \ldots \ldots$ & 1860 & Marignac ${ }^{5}$ & $\mathrm{~K}_{2} \mathrm{ZrF}_{6}: \mathrm{K}_{2} \mathrm{SO}_{4}$ & 4 & 90.03 \\
\hline & 1860 & Marignac & $\mathrm{K}_{2} \mathrm{ZTF}_{6}: \mathrm{ZrO}_{2}$ & 4 & 91.54 \\
\hline $7 \ldots \ldots \ldots$ & 1898 & Venable $^{6}$ & $\mathrm{ZrOCl}_{2 \cdot 3} \mathrm{H}_{2} \mathrm{O}: \mathrm{ZrO}_{2}$ & 10 & $90.8 \mathrm{I}$ \\
\hline & 1844 & Hermann ${ }^{7}$ & $\mathrm{ZrCl}_{4}: ?$ & $\mathbf{I}$ & 88.64 \\
\hline $9 \ldots \ldots \ldots$ & 1844 & Hermann ${ }^{7}$ & ${ }_{2} \mathrm{ZrOCl}_{2}, 9 \mathrm{H}_{2} \mathrm{O}:$ ? & 2 & 89.98 \\
\hline
\end{tabular}

We believe that little dependence is to be placed upon any of these figures. In the first three, zirconium sulfate is used. It is now well known that this substance cannot be freed from water without losing some sulfur trioxide. In addition it is difficult to prepare pure, especially avoiding the products of hydrolysis.

The series of determinations in No. 7 was carried out by one of us in I 898 and at the time of publication several sources of error were pointed out and a promise given of further work on the subject. Circumstances have prevented the fulfillment of the promise until now. The most serious

\footnotetext{
Clark, "Constants of Nature," p. 287 (19ro)

Ann. Physik., [I] 4, 126 (1825).

${ }^{3}$ Luind. Arsschrift, 18 (1881-2).

4. Chem. News, 60, 6, 17, 32 (1889).

5 Ann. chim. phys., [3] 60, 270 (1860).

- This JoURNal, 20, II9 (1898).

' $J$. prakt. Chem., 3I, 75 (1844).
} 